\title{
Quantification of User's Preference on Product Shapes Using Automobile as a Case Study
}

\author{
Nozomu MISHIMA ${ }^{1}$ and Taiga TOYOSHIMA \\ Akita University, Japan
}

\begin{abstract}
Product shapes have tight relations with user's preference. However, it is difficult for product designers to quantify the characteristics of product shapes and predict the preferred shapes. The author proposed a numerical method to quantify characteristics of smartphone shapes. Since the result showed a negative correlation between product impression strength versus user's preference, it was suggested that vigorous and weak impression is preferred. However, since smartphone has small shape variations, it is not a good example in validating the effectiveness of the method. In this research, a new case study for automobile shapes was carried out. As the result, it was suggested that the users can be categorized to some groups having different shape preferences. Since both groups preferred the shape with weak impression, it was suggested that people generally prefer simple and clear shapes for products. However, since the difference of the induction field value was small, this tendency should be examined through further study.
\end{abstract}

Keywords. User's preference, Semantic difference, Analytical hierarchy process, Induction field, Regression analysis

\section{Introduction}

In many products, product shapes must have tight relations with user's preference. Nowadays, since users' preferences are getting more and more various, some may require high functionalities and some may prefer sophisticated outlooks. The modern design activities should be transdisciplinary between functional and shape designs. However, it has been an unsolved problem for product designers to quantify characteristics of the shapes preferred by user and predict the user's preference on product shapes in advance. The recent trend is to combine numerical approaches with Kansei engineering methods. Many approaches [1-5] have been proposed for this purpose, in these days. The author also proposed a method in a previous paper [6] based on a numerical method named induction field $[7,8]$. The method was applied to quantify characteristics of smartphone shapes and calculate the correlation with the user's preference extracted by analytical hierarchy process [9].

Since the result showed a negative correlation between product impression strength versus user's preference, it was suggested that vigorous and week impression is relatively preferred by users. However, smartphone has small variations in terms of product shapes. In addition, people may guess the maker of the smartphone by the shapes used in the

\footnotetext{
${ }^{1}$ Corresponding Author, Mail: nmishima@gipc.akita-u.ac.jp.
} 
previous study. It will lead the users to consider about the product functions. Thus, smartphones might not be good examples in validating the effectiveness of the method. In this research, a new case study for automobile shapes was carried out. Since automobile is one of the products in which user's preference is affected by product shapes, it can be a good example for a case study. As for the automobile shapes there are previous achievements [10] in characterizing the product shapes. Since the automobile is one of the most important industrial products for modern societies, systemized approaches in designing shapes of the products may lead us to a promising outcome.

If this type of approach is successful, it will be possible to propose a more efficient and systemized design process that can also contribute in functional design of products. If the relation between product shapes and user's preference is clarified, it is also possible to determine which of shapes and functions should be focused on.

\section{Basics of induction field}

In the previous paper [6], the author has shown the procedure to apply the theory named induction field of vision [7, 8] in extracting the characteristics of product outlook quantitatively. The theory is to assume a "field" similar to an electrostatic field around a shape, when people see the shape. Such field is basically consisted of 2 major areas. Based on a Physiological observation, the human vison can be well-explained by stimulation of photoreceptor cells in the central area and suppression of the cells in the surrounding area. Such reaction was named side suppression. And people can recognize the edge of the object clearly by this reaction.

In order to simulate this reactions of photoreceptor cells numerically, expression like eq.(1) has been formulated. The shape of the object is expressed in the X-Y plane and the corresponding photoreceptor cells are expressed by $\xi-\psi$ plane. As it is expressed in the equation, when an optical stimulation comes to a certain point in the $\xi-\eta$ plane corresponding to the object shape in the X-Y plane, the nearby cells where difference of $X-\xi$ and $Y-\eta$ are small, are stimulated while surrounding cells will be suppressed.

$$
\begin{aligned}
S(x, y)= & \int_{\Delta S}\left\{\exp \left(-\frac{(\xi-x)^{2}+(\eta-y)^{2}}{2 * 5^{2}}\right)\right. \\
& \left.-\frac{5^{2}}{14^{2}} \times \exp \left(-\frac{(\xi-x)^{2}+(\eta-y)^{2}}{2 * 14^{2}}\right)\right\} L(\xi, \eta) d \xi d \eta
\end{aligned}
$$

$\mathrm{S}(x, y)$ : value of induction field at point $(x, y)$,

$L(\xi, \eta)$ : strength of the stimulation at point $(\xi, \eta)$

As for the practical value of the strength of the stimulation expressed by $L(\xi, \eta)$, bit map value of the corresponding bitmap file is used in the study. Since the bitmap data of a pixel is expressed as a discrete value from 0 to 255, the practical calculation value of eq.(1) and (2) will be discrete values. 


\section{Abstract result of the previous study}

\subsection{Case study on smartphone shapes}

In the previous study, using smartphone as the example, characteristics of product shapes was extracted by the induction field theory. 4 product shapes shown in Figure 1(a). through (d) were analyzed and the induction fields were calculated.

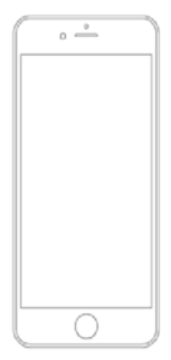

(a) Type A shape

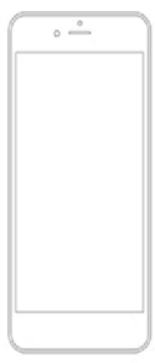

(c) Type C shape

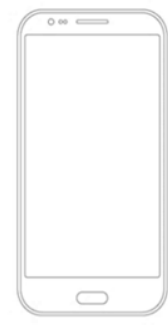

(b) Type B shape

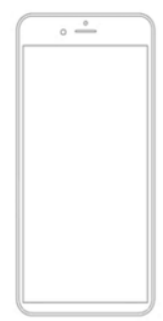

(d) Type D shape

Figure 1. Four different smartphone shapes

In analyzing the shape, two additional indices were introduced. Equation (2) and (3) are the formulation of two indices. $P E$ is expressing the total strength of the impression and $D I$ expresses the contrast of the shape.

$$
\begin{gathered}
P E=\iint_{0}^{+\infty}|S| \cdot A(|S|) d([S]) \\
D I=S_{\max }-S_{\min }
\end{gathered}
$$

$P E$ : potential value of the induction field

$D I$ : peak to peal value of the induction field

\subsection{User's preference of smartphone shapes}

In the previous study, characteristics of the product shapes were compared to the user's preference of the shape extracted by AHP (Analytical Hierarchy Process). In the study, 
three criterion of the shape evaluation were selected. Based on those three criterion indicated in Table 1, four smartphone shapes were evaluated through questionnaire. Table 2 is the total weight of four design plans and compositions of the plans regarding the three evaluation criterion.

Table 1. Three criterion of the smartphone shape and its' relative weight.

\begin{tabular}{|c|c|}
\hline Criterion & Relative weight \\
\hline Familiar & 0.25634 \\
\hline Easy-to-use & 0.54234 \\
\hline Sophisticated & 0.20132 \\
\hline
\end{tabular}

Table 2. Total weight of four design plans and compositions.

\begin{tabular}{|c|c|c|c|c|}
\hline Design plans & Familiar & Easy-to-use & Sophisticated & Total weight \\
\hline A & 0.12411 & 0.24242 & 0.065962 & 0.43249 \\
\hline B & 0.05382 & 0.13058 & 0.041518 & 0.22592 \\
\hline C & 0.04403 & 0.09037 & 0.040858 & 0.17525 \\
\hline D & 0.03437 & 0.07897 & 0.052985 & 0.16633 \\
\hline
\end{tabular}

\subsection{Correlation between characteristics of the shapes and user's preference}

The previous carried out regression analysis and calculate the regression coefficient with above-mentioned user's preferences and characteristics of the shapes. Table 3 shows the result of the regression analysis with PE and DI of the induction fields of the 4 designs and weight of each criterion.

The calculation showed negative correlations between, familiarity, easy-to-use and overall weight with the user's preferences. It basically means relatively weak and vigorous impressions are preferred by users.

Table 3. Correlations of evaluation criterion with induction fields

\begin{tabular}{|c|r|r|r|r|}
\hline Regression coefficient & \multicolumn{1}{|c|}{ Familiar } & Ease to use & \multicolumn{1}{c|}{ Sophisticated } & \multicolumn{1}{|c|}{ Overall weight } \\
\hline$P E$ & -0.529 & -0.449 & -0.196 & -0.461 \\
\hline$D I$ & -0.564 & -0.475 & -0.304 & -0.498 \\
\hline
\end{tabular}

\subsection{Points to be discussed}

Although the afore-mentioned result showed a rather clear negative correlations, the result should be carefully examined. Since there are some possibilities.

- The figures let the respondents image the actual product and the answers were affected by experiences and knowledges

- There is not a big difference in smartphone shapes and user's preferences are not affected by impression of the shapes

In order to answer these questions, another case study have been carried out. 


\section{A new case study regarding automobile shapes}

\subsection{Evaluation criteria}

In the previous study, negative correlations between overall impressions of shapes and user's preference were shown. However, since there is a research question that in case of smartphones, user's thought will not strongly affected by product shapes, a new case study was carried out in the study.

Automobile is a popular product that everyone can show one's preference and the product shapes affect the user's preference for sure. Based on the previous study [11], four criteria to evaluate automobile shapes were extracted. Those are "Sophisticated," "Sporty," "Familiar" and "Gentle."

\subsection{Weight of the criteria}

Table 4 is the result regarding the relative weight of criteria extracted through pair comparison of the criterion. In addition, it was found that it is possible to clarify user's preference better by the segmentation of respondents. The paper categorized the respondents into two groups. One is "Sporty-oriented" who puts emphasis on "sophisticated" and "sporty" rather than "familiar" and "gentle." The other is "Casualoriented" who has opposite preference. Table 5 and 6 are the typical analysis result of the respondents' answer who can be categorized to Sporty-oriented and Casual-oriented correspondingly.

Table 4. Average weight of four criteria

\begin{tabular}{|l|r|}
\hline Criteria & Relative weight \\
\hline Familiar & 0.249 \\
\hline Gentle & 0.141 \\
\hline Sophisticated & 0.229 \\
\hline Sporty & 0.381 \\
\hline
\end{tabular}

Table 5. Typical weight categorized to Sporty oriented group

\begin{tabular}{|l|r|}
\hline Criteria & Relative weight \\
\hline Familiar & 0.025 \\
\hline Gentle & 0.075 \\
\hline Sophisticated & 0.225 \\
\hline Sporty & 0.675 \\
\hline
\end{tabular}

Table 6. Typical weight categorized to Casual oriented group

\begin{tabular}{|l|r|}
\hline Criteria & Relative weight \\
\hline Familiar & 0.615 \\
\hline Gentle & 0.188 \\
\hline Sophisticated & 0.063 \\
\hline Sporty & 0.134 \\
\hline
\end{tabular}

\subsection{Preference on automobile shapes}

As it was mentioned before, knowledges and experiences of the actual products may affect the user's preference. Such effect should be eliminated to clarify user's preference purely based on product shapes. In order to avoid such affection, illustrations made from 
photos were used in the study. Information to specify the actual products such as make's logos were eliminated from the illustration. Figure 2.(a)-(h) are the illustrations.

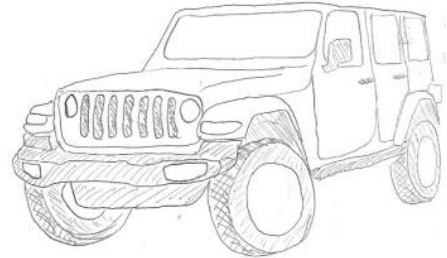

(a) SUV 1

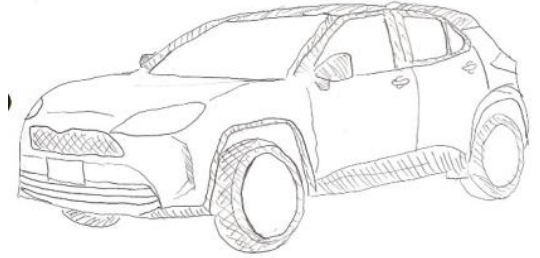

(b) SUV 2
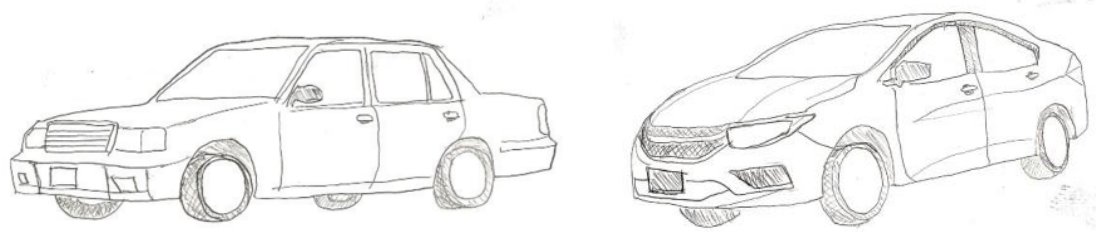

(c) sedan 1

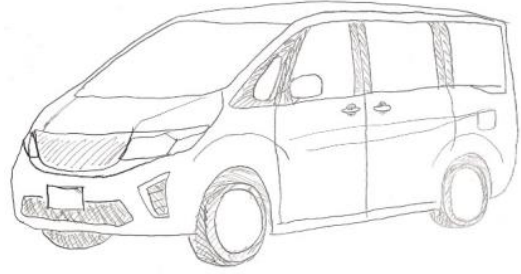

(e) minivan 1

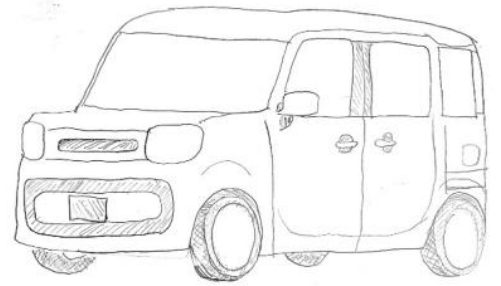

(g) wagon 1 (d) sedan 2

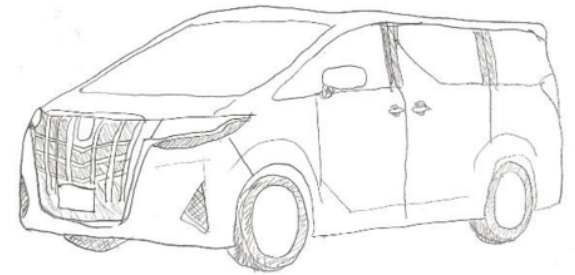

(f) minivan 2

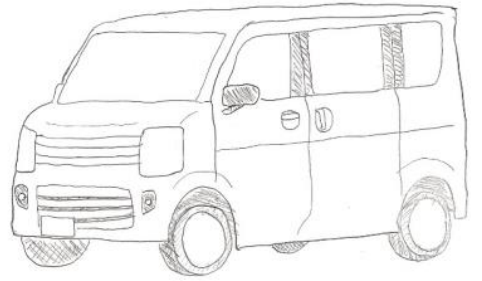

(b) wagon 2

Figure 2. Illustrations to quantify user's preference

Two user's group had rather different preferences on the illustrations. As for the second half of the survey, respondents' feeling on four pairs of automobile shapes regarding four evaluation criterion was investigated. Table 7 shows the result of the survey regarding relative weight two illustrations of four automobile types, for "Sportyoriented" users and "Casual-oriented" users separately. In other words, the table shows which one of two is preferred by which user group. Especially for SUV and minivan differences of preference for two groups were observed clearly. 
Table 7. Relative weights of 4 pairs of illustrations by two groups

\begin{tabular}{|l|c|c|c|c|c|c|c|c|}
\multicolumn{1}{c|}{} & \multicolumn{2}{c|}{ SUV } & \multicolumn{3}{c|}{ sedan } & \multicolumn{2}{c|}{ minivan } & \multicolumn{2}{c|}{ wagon } \\
\cline { 2 - 10 } \multicolumn{1}{c|}{1} & 2 & 1 & 2 & 1 & 2 & 1 & 2 \\
\hline Casual-oriented & 0.14 & 0.86 & 0.28 & 0.72 & 0.80 & 0.20 & 0.71 & 0.29 \\
\hline Sporty-oriented & 0.78 & 0.22 & 0.57 & 0.43 & 0.26 & 0.74 & 0.86 & 0.14 \\
\hline
\end{tabular}

\subsection{Induction field values}

While the two groups have rather different feelings against the illustrations, induction fields did not show clear tendencies. Induction fields of two illustrations of wagon had about 3\% differences. However, for other three automobile types, there were no clear differences of induction field values. Table 8 shows the PE and DI values of two illustrations of wagon.

Table 8. Induction fields of the illustrations of wagon

\begin{tabular}{|l|l|l|}
\hline & $P E$ & $D I$ \\
\hline 1 & $6.1533 \mathrm{E}+13$ & $4.1311 \mathrm{E}+05$ \\
\hline 2 & $6.3363 \mathrm{E}+13$ & $4.2437 \mathrm{E}+05$ \\
\hline
\end{tabular}

\subsection{Correlation of induction field and user's preferences}

As for the final steps of this procedure, correlation coefficients of two groups regarding the values of $\mathrm{PE}$ with relative weight of wagon illustration were calculated. As the result indicated in Table 9, for both groups, user's preference showed strong negative correlations with PE value extracted from the induction fields. Correlation with DI value was almost the same. However, it is premature to say it based on this calculation. Some further discussions are necessary.

Table 9. Correlation coefficients of 2 indices and PE value

\begin{tabular}{|c|c|c|}
\hline User groups & Casual-oriented & Sporty-oriented \\
\hline $\begin{array}{c}\text { Correlation } \\
\text { coefficient with } \mathrm{P} E\end{array}$ & -0.881 & -0.935 \\
\hline
\end{tabular}

\section{Discussions}

As it was mentioned in the previous chapter, it is too premature to say "there is a negative correlation between the overall strength of impression of the shape expressed by PE with user's preference." These are the reason to hesitate to jump to the conclusion.

- Although there were little differences regarding the two indices from induction field for automobile types except "wagon," clear differences of user's preference have been observed.

- The differences of user's preference may depend of the actual products that the illustrations suggest, rather than the pure impressions of the shapes. 
- Although the user's preference is affected by impression of the shape, but the proposed induction field does not express the impression correctly.

However, there are, on the other hand, some points that the authors think induction field is a hopeful method to extract the characteristics of the shape and can be helpful in clarifying and predicting user's preference on the product shapes.

- As it is shown in the formulation, calculation of the induction field is a process based on the bitmap data. Thus, in fact, the illustrations have such characteristics.

- Since the theory of induction field is based on psychological observations. In addition, the calculation procedure is similar to convolutional neural networks often used in deep learning of AI. So, it might be true that induction field expresses the human impression on shape exactly.

- Previous study also showed a negative correlation between impression strength and user's preference. It can be said that people do not like busy or messy shapes for product outlooks.

Whichever the fact is, it is necessary to evaluate the correlation between characteristics of the shapes and user's preference more precisely. In the aspect, below is the further research questions to be answered.

- It is necessary to survey user's preference by using shapes having rather different tendencies in tendencies of induction fields. By this effort, it will be possible to quantify correlations of the two items more clearly.

- Color might affect user's preference greatly. It is necessary to propose a method to quantify shape characteristics including color.

- The procedure used in this study is only applicable to two-dimensional shapes. An idea to treat three-dimensional shapes will be necessary.

\section{Conclusions}

In this paper, a procedure to extract characteristics of shapes by induction field theory and compare the characteristics to user's preference analyzed by AHP, by using illustrations of automobile as case studies.

It was found that respondents can be categorized to two major groups and the tendencies of preferences on automobile shapes were different corresponding to the group.

Only a pair of illustrations showed a little difference of induction field value, while the other 3 pairs showed little differences. And for the pair, negative correlation between overall impressions of the shapes and user's preference was shown. The fact suggests that people prefer weak and vigorous shapes for automobile, as well as for smartphones.

However, since still there are adore-mentioned problems in the procedure, it is necessary to proceed more precise examination regarding the issue. It is also necessary to analyze the people's perceptions against colors and three dimensional shapes. But, in future, this kind of effort to combine numerical analysis of product shapes and Kasie Engineering methods may reach to a more systematic and efficient stage of shape design of the products. 


\section{Acknowledgement}

This study is supported by Grants-in-Aid for Scientific Research, JP19K04137, provided by Japan Society for the Promotion of Science.

\section{References}

[1] F.G. Wu and C.Z. Yu, Parametric Design and Kansei engineering in Goblet Styling Design, Mathematical Problems in Engineering, 2020, Volume 2020, Article ID 8259698.

[2] Y, M, Chang and C. W. Chen, Kansei assessment on constituent elements and the overall interrelations in car steering wheel design, International Journal of Industrial Ergonomics, 2016, Vol.56, pp. 97-105.

[3] Q. Jiang and L. Peng, Kansei Image Evaluation of Teacup based on GA-ELM, In: 3rd International Conference on Mechatronics and Information Technology, 2018, pp. 246-253.

[4] M. D. Shieh, Y. Li and C. C. Yang, Comparison of multi-objective evolutionary algorithm in hybrid Kansei engineering system for product form design, Advanced Engineering Informatics, 2018, Vol. 36, pp. $31-42$.

[5] Z. Xiong, M. Liu, Y. Weng and Y. Liu, Research of Customer Product Form Preference based on Kansei Engineering, First International conference on Advanced Algorithm and Control Engineering, 2018, 1087, 062051.

[6] N. Mishima, Quantification of Shape Design and It's Correlation with User's Preference, Advances in Transdisciplinary Engineering, 2019, Vol. 10, pp. 252-260.

[7] I. Yokose, A Study on Character-Patterns Based Upon the Theory of Psychological Potential Field, Japanese Psychological Research, 1970, Vol. 12(1), pp. 18-25. (In Japanese)

[8] M. Nagaishi, A Consideration of Pattern Recognition using the Theory of Field of Induction on the Retina, IEICE technical report. Image engineering, 1995, 95(129), pp. 45-52.

[9] T. Saaty, Decision making with analytic hierarchy process, International Journal of Service Science, 2008, Vol.1, No.1, pp. 83-98.

[10] E. Ostrosi, J.B. Bluntzer, Z. Zhang and J. Stjepandić, Car style-holon recognition in computer-aided design, Journal of Computational Design and Engineering, 2019, Vol. 6, Issue 4, pp. 719-738.

[11] K. Inomata, G. Fujii, S. Hashimoto, T. Katahira, N. Nagata, T. Asano, K. Kawasaki and K. Nikata, Classification of Individuals based on Relationship between their Impression and Preference of Exterior Design of Vehicles, Transaction of Japan Society of Kansei Engineering, 2020, Vol.19, No. 2, pp. 223233. (In Japanese) 